EUROPEAN LABORATORY FOR PARTICLE PHYSICS

CERN-EP/98-010

23rd January 1998

\title{
Production of $f_{0}(980), f_{2}(1270)$ and $\phi(1020)$ in hadronic $\mathrm{Z}^{0}$ decay
}

\author{
The OPAL Collaboration
}

\begin{abstract}
Inclusive production of the $\mathrm{f}_{0}(980), \mathrm{f}_{2}(1270)$ and $\phi(1020)$ resonances has been studied in a sample of 4.3 million hadronic $\mathrm{Z}^{0}$ decays from the OPAL experiment at LEP. A coupled channel analysis has been used for the $\mathrm{f}_{0}$ in simultaneous fits to the resonances in inclusive $\pi^{+} \pi^{-}$and $\mathrm{K}^{+} \mathrm{K}^{-}$mass spectra. Fragmentation functions are reported for the three states. Total inclusive rates are measured to be $0.141 \pm 0.007 \pm 0.011 \mathrm{f}_{0}, 0.155 \pm 0.011 \pm 0.018 \mathrm{f}_{2}$ and $0.091 \pm 0.002 \pm 0.003$ $\phi$ mesons per hadronic $\mathrm{Z}^{0}$ decay. The production properties of the $\mathrm{f}_{0}$, including those in three-jet events, are compared with those of the $\mathrm{f}_{2}$ and $\phi$, and with the Lund string model of hadron production. All measurements are consistent with the hypothesis that the $\mathrm{f}_{0}(980)$ is a conventional q $\bar{q}$ scalar meson.
\end{abstract}

Submitted to European Physical Journal C: Particles and Fields 


\section{The OPAL Collaboration}

K. Ackerstaff ${ }^{8}$, G. Alexander ${ }^{23}$, J. Allison ${ }^{16}$, N. Altekamp ${ }^{5}$, K.J. Anderson ${ }^{9}$, S. Anderson ${ }^{12}$, S. Arcelli ${ }^{2}$, S. Asai ${ }^{24}$, S.F. Ashby ${ }^{1}$, D. Axen ${ }^{29}$, G. Azuelos ${ }^{18, a}$, A.H. Ball ${ }^{17}$, E. Barberio ${ }^{8}$, R.J. Barlow ${ }^{16}$, R. Bartoldus ${ }^{3}$, J.R. Batley ${ }^{5}$, S. Baumann ${ }^{3}$, J. Bechtluft ${ }^{14}$, T. Behnke ${ }^{8}$, K.W. Bell ${ }^{20}$, G. Bella ${ }^{23}$, S. Bentvelsen ${ }^{8}$, S. Bethke ${ }^{14}$, S. Betts ${ }^{15}$, O. Biebel ${ }^{14}$, A. Biguzzi ${ }^{5}$, S.D. Bird ${ }^{16}$, V. Blobel ${ }^{27}$, I.J. Bloodworth ${ }^{1}$, M. Bobinski ${ }^{10}$, P. Bock ${ }^{11}$, D. Bonacorsi ${ }^{2}$, M. Boutemeur ${ }^{34}$, S. Braibant ${ }^{8}$, L. Brigliadori ${ }^{2}$, R.M. Brown ${ }^{20}$, H.J. Burckhart ${ }^{8}$, C. Burgard ${ }^{8}$, R. Bürgin ${ }^{10}$, P. Capiluppi ${ }^{2}$, R.K. Carnegie ${ }^{6}$, A.A. Carter ${ }^{13}$, J.R. Carter ${ }^{5}$, C.Y. Chang ${ }^{17}$, D.G. Charlton ${ }^{1, b}$, D. Chrisman ${ }^{4}$, P.E.L. Clarke ${ }^{15}$, I. Cohen ${ }^{23}$, J.E. Conboy ${ }^{15}$, O.C. Cooke ${ }^{8}$, C. Couyoumtzelis ${ }^{13}$, R.L. Coxe ${ }^{9}$, M.Cuffiani ${ }^{2}$, S. Dado ${ }^{22}$, C. Dallapiccola ${ }^{17}$, G.M. Dallavalle ${ }^{2}$, R. Davis ${ }^{30}$, S. De Jong ${ }^{12}$, L.A. del Pozo ${ }^{4}$, A. de Roeck ${ }^{8}$, K. Desch ${ }^{8}$, B. Dienes ${ }^{33, d}$, M.S. Dixit ${ }^{7}$, M. Doucet ${ }^{18}$, E. Duchovni ${ }^{26}$, G. Duckeck ${ }^{34}$, I.P. Duerdoth ${ }^{16}$, D. Eatough ${ }^{16}$, P.G. Estabrooks ${ }^{6}$, E. Etzion ${ }^{23}$, H.G. Evans ${ }^{9}$, M. Evans ${ }^{13}$, F. Fabbri ${ }^{2}$, A. Fanfani ${ }^{2}$, M. Fanti ${ }^{2}$, A.A. Faust ${ }^{30}$, L. Feld ${ }^{8}$,

F. Fiedler ${ }^{27}$, M. Fierro ${ }^{2}$, H.M. Fischer ${ }^{3}$, I. Fleck ${ }^{8}$, R. Folman ${ }^{26}$, D.G. Fong ${ }^{17}$, M. Foucher ${ }^{17}$,

A. Fürtjes ${ }^{8}$, D.I. Futyan ${ }^{16}$, P. Gagnon ${ }^{7}$, J.W. Gary ${ }^{4}$, J. Gascon ${ }^{18}$, S.M. Gascon-Shotkin ${ }^{17}$, N.I. Geddes ${ }^{20}$, C. Geich-Gimbel ${ }^{3}$, T. Geralis ${ }^{20}$, G. Giacomelli ${ }^{2}$, P. Giacomelli ${ }^{4}$, R. Giacomelli ${ }^{2}$, V. Gibson ${ }^{5}$, W.R. Gibson ${ }^{13}$, D.M. Gingrich ${ }^{30, a}$, D. Glenzinski ${ }^{9}$, J. Goldberg ${ }^{22}$, M.J. Goodrick ${ }^{5}$,

W. Gorn ${ }^{4}$, C. Grandi' ${ }^{2}$ E. Gross ${ }^{26}$, J. Grunhaus ${ }^{23}$, M. Gruwé27 ${ }^{27}$ C. Hajdu ${ }^{32}$, G.G. Hanson ${ }^{12}$,

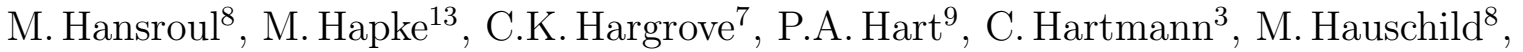
C.M. Hawkes ${ }^{5}$, R. Hawkings ${ }^{27}$, R.J. Hemingway ${ }^{6}$, M. Herndon ${ }^{17}$, G. Herten ${ }^{10}$, R.D. Heuer ${ }^{8}$, M.D. Hildreth ${ }^{8}$, J.C. Hill ${ }^{5}$, S.J. Hillier ${ }^{1}$, P.R. Hobson ${ }^{25}$, A. Hocker ${ }^{9}$, R.J. Homer ${ }^{1}$,

A.K.Honma $28, a$, D. Horváth ${ }^{32, c}$, K.R. Hossain ${ }^{30}$, R. Howard ${ }^{29}$, P. Hüntemeyer ${ }^{27}$,

D.E. Hutchcroft ${ }^{5}$, P. Igo-Kemenes ${ }^{11}$, D.C. Imrie ${ }^{25}$, K. Ishii ${ }^{24}$, A. Jawahery ${ }^{17}$, P.W. Jeffreys ${ }^{20}$, H. Jeremie ${ }^{18}$, M. Jimack ${ }^{1}$, A. Joly ${ }^{18}$, C.R. Jones ${ }^{5}$, M. Jones ${ }^{6}$, U. Jost ${ }^{11}$, P. Jovanovic ${ }^{1}$, T.R. Junk ${ }^{8}$, J. Kanzaki ${ }^{24}$, D. Karlen ${ }^{6}$, V. Kartvelishvili ${ }^{16}$, K. Kawagoe ${ }^{24}$, T. Kawamoto ${ }^{24}$, P.I. Kayal ${ }^{30}$, R.K. Keeler ${ }^{28}$, R.G. Kellogg ${ }^{17}$, B.W.Kennedy ${ }^{20}$, J. Kirk²9 , A. Klier ${ }^{26}$, S. Kluth ${ }^{8}$,

T. Kobayashi ${ }^{24}$, M. Kobel ${ }^{10}$, D.S. Koetke ${ }^{6}$, T.P. Kokott ${ }^{3}$, M. Kolrep ${ }^{10}$, S. Komamiya ${ }^{24}$, R.V.Kowalewski ${ }^{28}$, T. Kress ${ }^{11}$, P. Krieger ${ }^{6}$, J. von Krogh ${ }^{11}$, P. Kyberd ${ }^{13}$, G.D. Lafferty ${ }^{16}$, R. Lahmann ${ }^{17}$, W.P. Lai ${ }^{19}$, D. Lanske ${ }^{14}$, J. Lauber ${ }^{15}$, S.R. Lautenschlager ${ }^{31}$, I. Lawson ${ }^{28}$, J.G. Layter ${ }^{4}$, D. Lazic ${ }^{22}$, A.M. Lee ${ }^{31}$, E. Lefebvre ${ }^{18}$, D. Lellouch ${ }^{26}$, J. Letts ${ }^{12}$, L. Levinson ${ }^{26}$, B. List ${ }^{8}$, S.L. Lloyd ${ }^{13}$, F.K. Loebinger ${ }^{16}$, G.D. Long $^{28}$, M.J. Losty ${ }^{7}$, J. Ludwig ${ }^{10}$, D. Lui ${ }^{12}$,

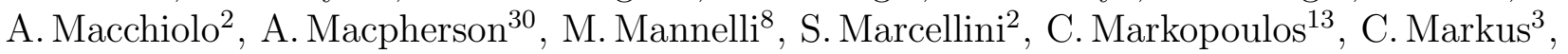
A.J. Martin ${ }^{13}$, J.P. Martin ${ }^{18}$, G. Martinez ${ }^{17}$, T. Mashimo ${ }^{24}$, P. Mättig ${ }^{26}$, W.J. McDonald ${ }^{30}$, J. McKenna ${ }^{29}$, E.A. Mckigney ${ }^{15}$, T.J. McMahon ${ }^{1}$, R.A. McPherson ${ }^{28}$, F. Meijers ${ }^{8}$, S. Menke $^{3}$, F.S. Merritt ${ }^{9}$, H. Mes ${ }^{7}$, J. Meyer ${ }^{27}$, A. Michelini ${ }^{2}$, S. Mihara ${ }^{24}$, G. Mikenberg ${ }^{26}$, D.J. Miller ${ }^{15}$, A. Mincer ${ }^{22, e}$, R. Mir ${ }^{26}$, W. Mohr ${ }^{10}$, A. Montanari ${ }^{2}$, T. Mori ${ }^{24}$, S. Mihara ${ }^{24}$, K. Nagai ${ }^{26}$, I. Nakamura ${ }^{24}$, H.A. Neal ${ }^{12}$, B. Nellen ${ }^{3}$, R. Nisius ${ }^{8}$, S.W. O’Neale ${ }^{1}$, F.G. Oakham ${ }^{7}$, F. Odorici ${ }^{2}$, H.O. Ogren ${ }^{12}$, A. $\mathrm{Oh}^{27}$, N.J. Oldershaw ${ }^{16}$, M.J. Oreglia ${ }^{9}$, S. Orito ${ }^{24}$, J. Pálinkás ${ }^{33, d}$, G. Pásztor ${ }^{32}$, J.R. Pater ${ }^{16}$, G.N. Patrick ${ }^{20}$, J. Patt ${ }^{10}$, R. Perez-Ochoa ${ }^{8}$, S. Petzold ${ }^{27}$, P. Pfeifenschneider ${ }^{14}$, J.E. Pilcher ${ }^{9}$, J. Pinfold ${ }^{30}$, D.E. Plane ${ }^{8}$, P. Poffenberger ${ }^{28}$, B. Poli ${ }^{2}$, A. Posthaus ${ }^{3}$, C. Rembser ${ }^{8}$, S. Robertson ${ }^{28}$, S.A. Robins ${ }^{22}$, N. Rodning ${ }^{30}$, J.M. Roney ${ }^{28}$,

A. Rooke ${ }^{15}$, A.M. Rossi ${ }^{2}$, P. Routenburg ${ }^{30}$, Y. Rozen $^{22}$, K. Runge $^{10}$, O. Runolfsson ${ }^{8}$, U.Ruppel ${ }^{14}$, D.R.Rust ${ }^{12}$, K. Sachs ${ }^{10}$, T. Saeki ${ }^{24}$, O. Sahr ${ }^{34}$, W.M.Sang ${ }^{25}$, E.K.G. Sarkisyan ${ }^{23}$, C.Sbarra ${ }^{29}$, A.D. Schaile ${ }^{34}$, O.Schaile ${ }^{34}$, F. Scharf ${ }^{3}$, P. Scharff-Hansen ${ }^{8}$, J. Schieck ${ }^{11}$, P.Schleper ${ }^{11}$, B. Schmitt ${ }^{8}$, S. Schmitt ${ }^{11}$, A. Schöning ${ }^{8}$, M. Schröder ${ }^{8}$, M. Schumacher ${ }^{3}$, C. Schwick ${ }^{8}$, W.G. $\operatorname{Scott}^{20}$, T.G.Shears ${ }^{8}$, B.C.Shen ${ }^{4}$, C.H. Shepherd-Themistocleous ${ }^{8}$, 
P. Sherwood ${ }^{15}$, G.P. Siroli ${ }^{2}$, A.Sittler ${ }^{27}$, A.Skillman ${ }^{15}$, A.Skuja ${ }^{17}$, A.M. $\operatorname{Smith}^{8}$, G.A.Snow ${ }^{17}$, R. Sobie ${ }^{28}$, S. Söldner-Rembold ${ }^{10}$, R.W.Springer ${ }^{30}$, M. Sproston ${ }^{20}$, K. Stephens ${ }^{16}$, J. Steuerer ${ }^{27}$, B. Stockhausen ${ }^{3}$, K. Stoll ${ }^{10}$, D. Strom ${ }^{19}$, R. Ströhmer ${ }^{34}$, P. Szymanski ${ }^{20}$, R. Tafirout ${ }^{18}$,

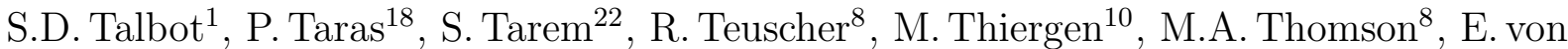
Törne $^{3}$, E. Torrence ${ }^{8}$, S. Towers ${ }^{6}$, I. Trigger ${ }^{18}$, Z. Trócsányi ${ }^{33}$, E. Tsur ${ }^{23}$, A.S. Turcot ${ }^{9}$, M.F. Turner-Watson ${ }^{8}$, I. Ueda ${ }^{24}$, P. Utzat ${ }^{11}$, R. Van Kooten ${ }^{12}$, P. Vannerem ${ }^{10}$, M. Verzocchi ${ }^{10}$, P. Vikas ${ }^{18}$, E.H. Vokurka ${ }^{16}$, H. Voss ${ }^{3}$, F. Wäckerle ${ }^{10}$, A. Wagner ${ }^{27}$, C.P. Ward ${ }^{5}$, D.R. Ward ${ }^{5}$, P.M. Watkins ${ }^{1}$, A.T. Watson ${ }^{1}$, N.K. Watson ${ }^{1}$, P.S. Wells ${ }^{8}$, N. Wermes ${ }^{3}$, J.S. White ${ }^{28}$, G.W.Wilson ${ }^{27}$, J.A.Wilson ${ }^{1}$, T.R. Wyatt ${ }^{16}$, S. Yamashita ${ }^{24}$, G. Yekutieli' ${ }^{26}$, V. Zacek ${ }^{18}$, D. Zer-Zion ${ }^{8}$

${ }^{1}$ School of Physics and Astronomy, University of Birmingham, Birmingham B15 2TT, UK 2Dipartimento di Fisica dell' Università di Bologna and INFN, I-40126 Bologna, Italy ${ }^{3}$ Physikalisches Institut, Universität Bonn, D-53115 Bonn, Germany

${ }^{4}$ Department of Physics, University of California, Riverside CA 92521, USA

${ }^{5}$ Cavendish Laboratory, Cambridge CB3 0HE, UK

${ }^{6}$ Ottawa-Carleton Institute for Physics, Department of Physics, Carleton University, Ottawa, Ontario K1S 5B6, Canada

${ }^{7}$ Centre for Research in Particle Physics, Carleton University, Ottawa, Ontario K1S 5B6, Canada

${ }^{8}$ CERN, European Organisation for Particle Physics, CH-1211 Geneva 23, Switzerland

${ }^{9}$ Enrico Fermi Institute and Department of Physics, University of Chicago, Chicago IL 60637, USA

${ }^{10}$ Fakultät für Physik, Albert Ludwigs Universität, D-79104 Freiburg, Germany

${ }^{11}$ Physikalisches Institut, Universität Heidelberg, D-69120 Heidelberg, Germany

${ }^{12}$ Indiana University, Department of Physics, Swain Hall West 117, Bloomington IN 47405, USA

${ }^{13}$ Queen Mary and Westfield College, University of London, London E1 4NS, UK

${ }^{14}$ Technische Hochschule Aachen, III Physikalisches Institut, Sommerfeldstrasse 26-28, D-52056 Aachen, Germany

${ }^{15}$ University College London, London WC1E 6BT, UK

${ }^{16}$ Department of Physics, Schuster Laboratory, The University, Manchester M13 9PL, UK

${ }^{17}$ Department of Physics, University of Maryland, College Park, MD 20742, USA

${ }^{18}$ Laboratoire de Physique Nucléaire, Université de Montréal, Montréal, Quebec H3C 3J7, Canada

${ }^{19}$ University of Oregon, Department of Physics, Eugene OR 97403, USA

${ }^{20}$ Rutherford Appleton Laboratory, Chilton, Didcot, Oxfordshire OX11 0QX, UK

${ }^{22}$ Department of Physics, Technion-Israel Institute of Technology, Haifa 32000, Israel

${ }^{23}$ Department of Physics and Astronomy, Tel Aviv University, Tel Aviv 69978, Israel

${ }^{24}$ International Centre for Elementary Particle Physics and Department of Physics, University of Tokyo, Tokyo 113, and Kobe University, Kobe 657, Japan

${ }^{25}$ Institute of Physical and Environmental Sciences, Brunel University, Uxbridge, Middlesex UB8 3PH, UK

${ }^{26}$ Particle Physics Department, Weizmann Institute of Science, Rehovot 76100, Israel

${ }^{27}$ Universität Hamburg/DESY, II Institut für Experimental Physik, Notkestrasse 85, D-22607 Hamburg, Germany 
${ }^{28}$ University of Victoria, Department of Physics, P O Box 3055, Victoria BC V8W 3P6, Canada ${ }^{29}$ University of British Columbia, Department of Physics, Vancouver BC V6T 1Z1, Canada

${ }^{30}$ University of Alberta, Department of Physics, Edmonton AB T6G 2J1, Canada

${ }^{31}$ Duke University, Dept of Physics, Durham, NC 27708-0305, USA

${ }^{32}$ Research Institute for Particle and Nuclear Physics, H-1525 Budapest, P O Box 49, Hungary

${ }^{33}$ Institute of Nuclear Research, H-4001 Debrecen, P O Box 51, Hungary

${ }^{34}$ Ludwigs-Maximilians-Universität München, Sektion Physik, Am Coulombwall 1, D-85748 Garching, Germany

${ }^{a}$ and at TRIUMF, Vancouver, Canada V6T 2A3

${ }^{b}$ and Royal Society University Research Fellow

${ }^{c}$ and Institute of Nuclear Research, Debrecen, Hungary

${ }^{d}$ and Department of Experimental Physics, Lajos Kossuth University, Debrecen, Hungary

${ }^{e}$ and Department of Physics, New York University, NY 1003, USA 


\section{Introduction}

Inclusive production of mesons and baryons in $\mathrm{Z}^{0}$ decay has been studied extensively [1], and the results have provided valuable input to the theory and phenomenology of parton hadronization. Production of the $\mathrm{f}_{0}(980)$ is of particular interest because although it is well established experimentally as a scalar $\left(\mathrm{J}^{\mathrm{PC}}=0^{++}\right)$state, its precise nature has long been uncertain. Indeed, there is still vigorous debate [2, 3] about the identity of the mesons which comprise the lowest lying scalar nonet of flavour SU(3). Compared to the expectations for a conventional meson at a mass of around $1 \mathrm{GeV}$, the $\mathrm{f}_{0}$ has a markedly small total width, a relatively large coupling to $\mathrm{K} \overline{\mathrm{K}}$, and a partial width to $\gamma \gamma$ which is an order of magnitude below theoretical expectation.

A number of suggestions have been made as to the nature of the $\mathrm{f}_{0}$. Jaffe and Johnson [4] performed a bag model calculation to suggest that it could be a "cryptoexotic" qq $\bar{q} \bar{q}$ state. Weinstein and Isgur [5], using a potential model of qq $\bar{q} \bar{q}$ states, showed that the $\mathrm{f}_{0}$ could be explained as a loosely bound $\mathrm{K} \overline{\mathrm{K}}$ system, a so-called $\mathrm{K} \overline{\mathrm{K}}$ molecule. Gribov [6, 7] has proposed a theory of confinement in QCD, in which the $\mathrm{f}_{0}$ plays the role of a novel "vacuum scalar" state, a bound state of a quark and antiquark with negative kinetic energy, interacting repulsively to give a state of positive total energy. Ishida et al. [8 have proposed an interpretation as a hybrid meson with a massive constituent gluon, while a scalar glueball has been suggested by Robson [9]. An analysis by Close and Amsler [10] of the scalar states suggests that the most likely candidate for the lowest-lying scalar glueball is the $\mathrm{f}_{0}(1500)$; in this model, the $\mathrm{f}_{0}(980)$ is a left-over state which cannot be accommodated in the scalar nonet. Lattice QCD calculations 11] also find the lightest scalar glueball mass to be around $1.5 \mathrm{GeV}$ so that the glueball interpretation of the $\mathrm{f}_{0}$ is now out of favour. Törnqvist [12], using a unitarized quark model to analyse all of the identified states in the $0^{++}$sector, has concluded however that the $\mathrm{f}_{0}$ can fit in as a conventional $\mathrm{q} \overline{\mathrm{q}}$ meson in the lowest $0^{++}$multiplet. Similarly, an analysis by Zou and Bugg [13] of all available high-statistics $\pi^{+} \pi^{-}$and $\mathrm{K} \overline{\mathrm{K}}$ scattering data concluded that the $\mathrm{f}_{0}$ could be interpreted as a meson, and recent work by Anisovich and Sarantsev [14] has come to the same conclusion.

One aim of the present study is to look for features of $\mathrm{f}_{0}$ production in $\mathrm{Z}^{0}$ decay which may help to elucidate its nature. The vacuum scalar states of Gribov's theory are expected to be compact objects with distinctive production properties, and suggestions have been made by Close et al. [7] for a number of experimental tests. In $\mathrm{Z}^{0}$ decay, the signature would be a relatively larger yield in low multiplicity events and in events where the $\mathrm{f}_{0}$ is isolated in rapidity. If, on the other hand, the $\mathrm{f}_{0}$ has a significant gluonic content, its production could be enhanced in gluon jets. In contrast, if it is principally a conventional meson, its production properties may be unremarkable when compared to those of other similar states. There are presently no predictions for production rates of qq $\bar{q} \bar{q}$ states or $\mathrm{K} \overline{\mathrm{K}}$ molecules in $\mathrm{Z}^{0}$ decay.

Two approaches are taken in the present analysis: a comparison of the features of $\mathrm{f}_{0}$ production with those of two neutral isoscalar mesons, and a comparison with the JETSET 7.4 [15] implementation of the Lund string model of hadronization [16], within which the $\mathrm{f}_{0}$ is treated as a conventional meson. The features of $\mathrm{f}_{0}$ production are measured along with those of the $\mathrm{f}_{2}(1270)$ and the $\phi(1020)$ mesons. The latter is close in mass to the $\mathrm{f}_{0}$, while the former is an established meson with the $\mathrm{q} \overline{\mathrm{q}}$ in a ${ }^{3} \mathrm{P}_{2}$ state. In the conventional meson interpretation, the $\mathrm{f}_{0}$ 
would be a ${ }^{3} \mathrm{P}_{0}$ state. Since the mass difference is relatively small, the $\mathrm{f}_{0}$ and $\mathrm{f}_{2}$ may then be expected to have similar production properties. The Lund string model, as implemented in the JETSET 7.4 Monte Carlo program, is highly successful in describing many features of hadronic $\mathrm{Z}^{0}$ decay events. These features include global event properties related to the perturbative phase of the initial parton shower, as well as details of the production of many different species of hadrons during the nonperturbative hadronization phase. In short, the model provides a well-tested and reliable picture of particle production in hadronic $\mathrm{Z}^{0}$ decays [17]. Within the JETSET model, the $\mathrm{f}_{0}$ is treated as a scalar meson composed of $u \bar{u}$ and $\mathrm{d} \overline{\mathrm{d}}$ pairs, and the relative production rates of ${ }^{3} \mathrm{P}_{0}$ and ${ }^{3} \mathrm{P}_{2}$ mesons are determined by a variable parameter. $\mathrm{A}$ comparison of the data and the results of JETSET could therefore provide information on the nature of the $f_{0}$.

The only reported measurements of the $\mathrm{f}_{0}$ and $\mathrm{f}_{2}$ at LEP were made by DELPHI as part of a general study of light resonances [18 using relatively low statistics. The fragmentation functions and total rates were reported over restricted ranges of momentum. In a similar study of resonance production by ALEPH [19], the $\mathrm{f}_{0}$ was included in fits to $\pi^{+} \pi^{-}$mass spectra, but no rates were reported. Because the $\mathrm{f}_{0}$ has a significant coupling to $\mathrm{K} \overline{\mathrm{K}}$ and peaks below threshold for this channel, its $\pi^{+} \pi^{-}$mass spectrum will not in general exhibit the simple BreitWigner shape assumed in these two previous analyses. In the present study, a coupled-channel analysis is done, including $\pi^{+} \pi^{-}$and $\mathrm{K}^{+} \mathrm{K}^{-}$data, in which proper account is taken of the opening of the $\mathrm{K} \overline{\mathrm{K}}$ channel in $\mathrm{f}_{0}$ decay.

\section{The OPAL detector and data samples}

The OPAL detector is described in [20]. For the present analysis, the most important components were the central tracking chambers which consist of two layers of silicon microvertex detectors [21], a high-precision vertex drift chamber, a large-volume jet chamber, and a set of drift chambers (the $z$-chambers) which measure the coordinates of tracks along the direction of the beam. The OPAL coordinate system is defined with the $z$-axis following the electron beam direction; the polar angle $\theta$ is defined relative to this axis, and $r$ and $\phi$ are the usual cylindrical polar coordinates. The central chambers lie within a homogeneous axial magnetic field of $0.435 \mathrm{~T}$. Charged particle tracking is possible over the range $|\cos \theta|<0.98$ for the full range of azimuthal angles. The OPAL jet chamber is capable of measuring specific energy loss, $\mathrm{d} E / \mathrm{d} x$, with a resolution, $\sigma(\mathrm{d} E / \mathrm{d} x) /(\mathrm{d} E / \mathrm{d} x)$, of $3.5 \%$ for well-reconstructed, high-momentum tracks in hadronic events 22].

The present analysis used the full OPAL sample of 4.3 million hadronic $\mathrm{Z}^{0}$ decays recorded at LEP 1 between 1990 and 1995. To correct for losses due to the acceptance and efficiency of the experiment and the selection procedures, and also to provide signal and background shapes for fits to the data mass spectra, 6 million Monte Carlo events were used, which had been generated using JETSET 7.4 and processed through a full simulation of the experiment [23] and the data reconstruction and analysis. This 'detector-level' Monte Carlo sample was also used for comparison with various features of the experimental data. The JETSET version was tuned [24] using OPAL data on event shape distributions, fragmentation functions of $\pi^{ \pm}, \mathrm{K}^{ \pm}$, $\mathrm{p} / \overline{\mathrm{p}}$ and $\Lambda$, and LEP data on total inclusive multiplicities for 26 identified hadron species. 
A detailed description of the selection of hadronic $\mathrm{Z}^{0}$ decay events in OPAL is given in 25]. For the present analysis, tracks in the selected events were required to have: a minimum momentum transverse to the beam direction of $150 \mathrm{MeV} / c$; a maximum momentum of $1.07 \times$ $E_{\text {beam }}$, based on the momentum resolution of the detector; a distance of closest approach to the interaction point less than $5 \mathrm{~cm}$ in the plane orthogonal to the beam direction, and the corresponding distance along the beam direction less than $40 \mathrm{~cm}$; a first measured point within a radius of $75 \mathrm{~cm}$ from the vertex; and at least 20 hits available for measurement of specific energy loss, $\mathrm{d} E / \mathrm{d} x$.

Kaons and pions were identified using the $\mathrm{d} E / \mathrm{d} x$ measurements. For each track, a $\chi^{2}$ probability (weight) was formed for each of the stable particle hypotheses (e, $\mu, \pi, \mathrm{K}$ and p). A track was identified as a pion or a kaon if the appropriate weight was above $5 \%$ and was larger than the weight for each of the other stable hadron hypotheses. Between momenta of 0.8 and $2.0 \mathrm{GeV} / c$, the $\pi, \mathrm{K}$ and $\mathrm{p}$ bands overlap in $\mathrm{d} E / \mathrm{d} x$, leading to considerable ambiguity among hypotheses. Therefore, no tracks were identified as kaons in this momentum range although, since most particles are pions, pion identification was still allowed.

With the event and track selection cuts previously described, $\mathrm{f}_{0}$ and $\mathrm{f}_{2}$ decaying via $\pi^{+} \pi^{-}$ were identified with an efficiency of around $40 \%$ over the whole momentum range. The $\phi \rightarrow$ $\mathrm{K}^{+} \mathrm{K}^{-}$efficiency typically varied between 15 and $20 \%$, although in the $\phi$ momentum range from 1.4 to $3.6 \mathrm{GeV} / c$ it fell below $5 \%$ because of the $\mathrm{d} E / \mathrm{d} x$ cross-over region. The mass resolution at $1 \mathrm{GeV}$ varied with momentum, from 15 to $20 \mathrm{MeV}$ for $\pi^{+} \pi^{-}$and from 2.5 to $4.5 \mathrm{MeV}$ for $\mathrm{K}^{+} \mathrm{K}^{-}$.

\section{Data analysis}

\subsection{Inclusive two-particle mass spectra}

For simultaneous fits to $\pi^{+} \pi^{-}$and $\mathrm{K}^{+} \mathrm{K}^{-}$mass spectra in both the real and simulated data samples, spectra were formed for inclusive $\pi^{+} \pi^{-}, \pi^{ \pm} \pi^{ \pm}$and $\mathrm{K}^{+} \mathrm{K}^{-}$systems in bins of each of the following variables:

- scaled momentum, $x_{p}\left(=p / E_{\text {beam }}\right)$, of the two-particle systems (nine bins)

- rapidity, $y$, of the two-particle systems with respect to the event thrust axis (six bins)

- rapidity gap, $\Delta y$, between the two-particle systems and the closest single charged particle (six bins)

- multiplicity, $n_{\mathrm{ch}}$, of charged tracks in the event (six bins)

The last three of these variables were chosen specifically for tests of the nature of the $f_{0}$, as discussed in section 1. To account for a large part of the combinatorial backgrounds in $\pi^{+} \pi^{-}$, mass spectra were formed by subtracting the $\pi^{ \pm} \pi^{ \pm}$spectra from those for $\pi^{+} \pi^{-}$. Because the combinatorial backgrounds in $\mathrm{K}^{+} \mathrm{K}^{-}$were relatively small, and Bose-Einstein correlations could 
affect the $\mathrm{K}^{ \pm} \mathrm{K}^{ \pm}$spectra near threshold, no subtraction was done for the $\mathrm{K}^{+} \mathrm{K}^{-}$mass spectra. For the Monte Carlo sample, separate spectra were also made for the most important states contributing to the mass spectra, using information on the origin of each track at the generator level.

\subsection{Selection of three-jet events}

To investigate possible differences between production in quark and gluon jets, the Durham jet finder 27] was used to identify a sample of three-jet events. The cut-off value $y_{\text {cut }}$ was set to 0.005, and the jet-finding was done using charged tracks. For each candidate three-jet event, the angle between the two lowest-energy jets was required to be greater than $30^{\circ}$, and in order to ensure well-reconstructed, planar events, the sum of the interjet angles was required to be larger than $358^{\circ}$. The jet energies were then reconstructed using the interjet angles, assuming massless kinematics. Each jet was required to contain at least two charged particles and more than $5 \mathrm{GeV}$ of energy. With these cuts, $24 \%$ of all events were selected as three-jet events. Monte Carlo studies have shown that in such events, $95 \%$ of the highest-energy jets are due to quarks, while the lowest-energy jet is a gluon jet with an approximately $80 \%$ probability. Mass spectra were formed, using only tracks assigned to the same jet, in three bins of $E / E_{\text {jet }}$ where $E$ is the energy of the two-particle system and $E_{\text {jet }}$ is the energy of the jet.

\subsection{Fit procedures}

For each bin of the above kinematic variables, the $\pi^{+} \pi^{-}$and $\mathrm{K}^{+} \mathrm{K}^{-}$mass spectra were fitted simultaneously, using a minimum $\chi^{2}$ fit, to a sum of contributions given by:

$$
\begin{gathered}
f\left(m_{\pi^{+} \pi^{-}}\right)=a_{\mathrm{f}_{0}}^{\pi^{+} \pi^{-}} \mid A_{\mathrm{f}_{0}}\left(\left.m_{\pi \pi)}\right|^{2}+a_{\mathrm{f}_{2}} \mathrm{BW}_{\mathrm{f}_{2}}+a_{\mathrm{bgd}}^{\pi^{+} \pi^{-}} B_{\pi^{+} \pi^{-}}\right. \\
f\left(m_{\mathrm{K}^{+} \mathrm{K}^{-}}\right)=a_{\mathrm{f}_{0}}^{\mathrm{K}^{+} \mathrm{K}^{-}}\left|A_{\mathrm{f}_{0}}\left(m_{\mathrm{KK}}\right)\right|^{2}+a_{\phi} \mathrm{BW}_{\phi}+a_{\mathrm{bgd}}^{\mathrm{K}^{+} \mathrm{K}^{-}} B_{\mathrm{K}^{+} \mathrm{K}^{-}}
\end{gathered}
$$

In equations (11) and (2), the $a$ terms represent the intensities to be fitted, $A_{\mathrm{f}_{0}}$ is the amplitude for $\mathrm{f}_{0}(980)$, BW are Breit-Wigner functions, and the $B$ represent background functions. The $a_{\mathrm{f}_{0}}$ are related by

$$
a_{\mathrm{f}_{0}}^{\mathrm{K}^{+} \mathrm{K}^{-}}=0.75 \frac{e_{\mathrm{K}^{+} \mathrm{K}^{-}}}{e_{\pi^{+} \pi^{-}}} a_{\mathrm{f}_{0}}^{\pi^{+} \pi^{-}}
$$

where $e_{\mathrm{K}^{+} \mathrm{K}^{-}} / e_{\pi^{+} \pi^{-}}$is the ratio of the efficiency to reconstruct $\mathrm{f}_{0}$ in $\mathrm{K}^{+} \mathrm{K}^{-}$relative to $\pi^{+} \pi^{-}$ (which varies with the bin of the kinematic variable), and the factor 0.75 comes from ClebschGordan coefficients. Following Flatté [26], the coupled-channel amplitudes for $\mathrm{f}_{0}(980)$ decay via $\pi \pi$ and KK were taken to be:

$$
A_{\mathrm{f}_{0}}\left(m_{\pi \pi}\right)=\frac{m_{0} \sqrt{\Gamma_{\pi \pi}}}{m_{0}^{2}-m_{\pi \pi}^{2}-i m_{0}\left(\Gamma_{\pi \pi}+\Gamma_{\mathrm{KK}}\right)}
$$




$$
A_{\mathrm{f}_{0}}\left(m_{\mathrm{KK}}\right)=\frac{m_{0} \sqrt{\Gamma_{\mathrm{KK}}}}{m_{0}^{2}-m_{\mathrm{KK}}^{2}-i m_{0}\left(\Gamma_{\pi \pi}+\Gamma_{\mathrm{KK}}\right)}
$$

Here $m_{0}$ is the resonance mass, and the partial widths $\Gamma$ are related to the coupling constants $g$ via

$$
\Gamma_{\pi \pi}=g_{\pi} \sqrt{\frac{m_{\pi \pi}^{2}}{4}-m_{\pi}^{2}} \quad \text { and } \quad \Gamma_{\mathrm{KK}}=g_{\mathrm{K}} \sqrt{\frac{m_{\mathrm{KK}}^{2}}{4}-m_{\mathrm{K}}^{2}}
$$

The fact that $\Gamma_{\mathrm{KK}}$ is imaginary below KK threshold leads to distortion of the $\pi \pi$ mass spectrum from a simple Breit-Wigner shape. Interference between $\mathrm{f}_{0}$ and $\pi^{+} \pi^{-}$backgrounds in $\mathrm{Z}^{0}$ decay should be negligible and was not included in the fits. In determining the meson rates, the possible presence of other f-meson resonances coupling to $\pi^{+} \pi^{-}$has been neglected.

The experimental mass resolution was treated differently for the three states. The $\mathrm{f}_{0}$ intensities derived from equation (3) were folded with the detector resolution separately for each bin of $x_{p}, y, \Delta y, n_{\mathrm{ch}}$ and $E / E_{\text {jet }}$. For the broad $\mathrm{f}_{2}$, a relativistic D-wave Breit-Wigner was used, with mass and width fixed to the Particle Data Group (PDG) values [3]. For the $\phi$, where experimental mass resolution is particularly important, the shapes for the fits were taken from the detector-level output of the simulation, accounting automatically for variations of the mass resolution. The background shapes for the fits were also taken from the detector-level Monte Carlo: for $\pi^{+} \pi^{-}$, all pairs identified as $\pi^{+} \pi^{-}$were taken, except those from the $\mathrm{f}_{0}$ and $\mathrm{f}_{2}$; and for the $\mathrm{K}^{+} \mathrm{K}^{-}$, all identified combinations were used except those due to $\mathrm{f}_{0}$ and $\phi$. In the fits, the background shapes were given additional freedom, with the $\pi^{+} \pi^{-}$contribution multiplied by a term $\left(m_{\pi^{+} \pi^{-}}^{\alpha_{1}}+\alpha_{2} m_{\pi^{+} \pi^{-}}^{\alpha_{3}}\right)$ and the $\mathrm{K}^{+} \mathrm{K}^{-}$shape by $\left(1+\alpha_{4} m_{\mathrm{K}^{+} \mathrm{K}^{-}}\right)$, with the $\alpha$ being variable parameters. The $\rho^{0}$ resonance in the JETSET simulation is truncated at $\pm 2.5 \Gamma$; to allow for this, the $\rho^{0}$ intensity above $1.15 \mathrm{GeV}$ was taken as a straight-line extrapolation from the contribution at $1.15 \mathrm{GeV}$ falling to zero at $1.5 \mathrm{GeV}$.

The mass ranges of the fits were 0.82 to $1.5 \mathrm{GeV}$ for $\pi^{+} \pi^{-}$, and from threshold to $1.18 \mathrm{GeV}$ for $\mathrm{K}^{+} \mathrm{K}^{-}$, these limits being chosen mainly to avoid the $\rho^{0}$ peak region in $\pi^{+} \pi^{-}$and the region of its reflection in $\mathrm{K}^{+} \mathrm{K}^{-}$(caused by particle misidentification). Figure 1 shows as an example the sum of the fitted mass spectra over the range $x_{p}>0.14$, indicating clearly the signals due to the three resonances.

In fits to the total mass spectra for $x_{p}>0.14$ with the $\mathrm{f}_{0}$ parameters allowed to vary, the mass was well-constrained while the couplings $g$ were poorly determined: $m_{0}=0.957 \pm 0.006 \mathrm{GeV}$, $g_{\pi}=0.09 \pm 0.40$ and $g_{\mathrm{K}}=0.97 \pm 0.82$. This is essentially due to the limited mass resolution and the large backgrounds in the $\pi^{+} \pi^{-}$channel; the $\mathrm{K}^{+} \mathrm{K}^{-}$data are relatively insensitive to the $\mathrm{f}_{0}$ parameters. The fitted mass value is in good agreement with that obtained in the highstatistics analysis made by Zou and Bugg [13] of $\pi \pi-\mathrm{K} \overline{\mathrm{K}}$ elastic scattering phase shifts and $\pi \pi$ and $\mathrm{K} \overline{\mathrm{K}}$ mass spectra from central production in pp collisions. The $m_{0}$ and $g$ values were therefore fixed to the Zou and Bugg values, $m_{0}=0.9535 \mathrm{GeV}, g_{\pi}=0.111$ and $g_{\mathrm{K}}=0.423$. These couplings correspond to a branching ratio, $\mathrm{BR}\left(\mathrm{f}_{0} \rightarrow \pi \pi\right)=0.80$, in agreement with the PDG value. Nevertheless, there is much experimental and theoretical uncertainty in the resonance parameters appropriate for the $\mathrm{f}_{0}$. Indeed the PDG quotes the total width as 40 to $100 \mathrm{MeV}$. The results should therefore be taken as model-dependent measurements, which assume the Flatté parametrization with the parameter values of Zou and Bugg, and no interference with background. Fits to the $\pi^{+} \pi^{-}$and $\mathrm{K}^{+} \mathrm{K}^{-}$spectra are shown in figures $\mathrm{Q}$ and $\mathrm{f}$ for six bins of 
$x_{p}$; it can be seen from the figures that the parametrization works well in the $\mathrm{Z}^{0}$ decay data over the entire kinematic range.

\subsection{Fragmentation functions and total rates}

The rates obtained from the fits in bins of $x_{p}$ are given in table 1. Figure 1 shows these measured fragmentation functions, $\left(1 / \sigma_{\mathrm{h}}\right) \mathrm{d} \sigma / \mathrm{d} x_{p}$ (where $\sigma_{\mathrm{h}}$ is the total hadronic cross section), along with curves from the JETSET 7.4 Monte Carlo generator, normalized for each particle to the measured total rate seen in the data. Only statistical errors are shown for the $\mathrm{f}_{0}$ and $\mathrm{f}_{2}$; the dominant systematic errors are correlated over the $x_{p}$ bins and are discussed below in section 3.5. There is clearly good agreement for all three particles between the shapes of the momentum distributions in the Monte Carlo model and those in the data.

The total inclusive rates have been measured, by integrating the fragmentation functions, to be:

$$
\begin{array}{lc}
0.141 \pm 0.007 \pm 0.011 & \mathrm{f}_{0}(980) \\
0.155 \pm 0.011 \pm 0.018 & \mathrm{f}_{2}(1270) \\
0.091 \pm 0.002 \pm 0.003 & \phi(1020)
\end{array}
$$

per hadronic $\mathrm{Z}^{0}$ decay. The first errors are statistical and the second systematic (discussed below). These rates compare with values of $0.154 \pm 0.025 \mathrm{f}_{0}$ and $0.240 \pm 0.061 \mathrm{f}_{2}$ mesons, obtained by Böhrer [1] by extrapolating the DELPHI measurements [18] to the full range of $x_{p}$. The measurement for the $\phi$ is in agreement with previous OPAL [28] and DELPHI [30] results, but is three standard deviations below an ALEPH 229 measurement. The present $\phi$ measurement has the smallest statistical and systematic errors.

\subsection{Systematic errors}

Table 2 gives a summary of systematic errors on the meson rates. To estimate systematic errors for the $\mathrm{f}_{0}$, the uncertainties in the resonance mass and the couplings were considered. The value of $m_{0}$ was varied from 0.951 to $0.963 \mathrm{GeV}$ (the range obtained when the mass was allowed to be free in the fits) while keeping the coupling constants fixed. The maximum total variation, $2.5 \%$, was taken as the systematic error. Next $m_{0}$ and $g_{\mathrm{K}}$ were fixed and the coupling $g_{\pi}$ was allowed to vary freely in the fit. This gave a $7 \%$ change in the total rate, which was conservatively taken as the systematic error from this source. (If, instead, the errors in the $\pi \pi$ branching ratio from the PDG are used, the systematic error would be $3 \%$.) Finally, $m_{0}$ and $g_{\pi}$ were fixed and $g_{\mathrm{K}}$ was allowed to vary. The $\mathrm{f}_{0}$ rate changed by much less than the statistical error, while the $\phi$ rate increased by $1.5 \%$; this was assigned as a systematic error on the $\phi$ measurement. Systematic errors from uncertainties in Monte Carlo modelling of the track cuts contributes $1.4 \%$ 28] for resonances decaying to two charged particles. The overall systematic error on the $\mathrm{f}_{0}$ measurements was therefore $7.6 \%$.

For the $\mathrm{f}_{2}$ measurements, the main sources of systematic uncertainty also come from the resonance parametrization, although in this case it is safe to assume a normal Breit-Wigner 
resonance. To take account of possible long tails in the line shape beyond the upper limit of the fits to the mass spectra, the relativistic D-wave Breit-Wigner used for the fits was integrated out to $2.2 \mathrm{GeV}$, five full widths above the nominal peak position. This resulted in an increase of $30 \%$ in the total intensity over the fitted value. Since the shape of such a resonance is uncertain so far from the pole position, one half of this extra contribution was added to each measurement of the differential cross section (the results in table 1 include these corrections), and a systematic error of $30 \% / \sqrt{12}$ was assigned. The fits were repeated, varying the $\mathrm{f}_{2}$ mass and width by one standard deviation (using the PDG values) above and below their nominal values. The maximum change in the measured rate, $6 \%$, was assigned as a systematic error. To account for the high mass tail of the $\rho^{0}$ above $1.15 \mathrm{GeV}$, the fits were repeated assuming two extreme possibilities: the default JETSET simulation, with the shape truncated at $1.15 \mathrm{GeV}$; and a constant contribution set to the level in the simulation just below $1.15 \mathrm{GeV}$. The maximum change, $3.2 \%$, was taken as the systematic error. With an error of $3.1 \%$ on the branching ratio to $\pi^{+} \pi^{-}$and $1.4 \%$ from the modelling of the track cuts, the overall systematic error on the $\mathrm{f}_{2}$ measurements was therefore $11.6 \%$.

Systematic errors due to the simulation of the energy loss were measured by varying the assumed mean values of the theoretical $\mathrm{d} E / \mathrm{d} x$ distributions for a given particle hypothesis, and the assumed resolution on the energy loss measurements. Studies of well-identified pions from $\mathrm{K}_{\mathrm{S}}^{0}$ decays, protons from $\Lambda$ decays and kaons from $\mathrm{D}^{0}$ decays were used to place limits on the maximum possible deviations of these quantities, and the analysis was repeated, with the $\mathrm{d} E / \mathrm{d} x$ weights of the tracks being recalculated each time. Systematic errors were assigned as the maximum measured deviations from the standard fit values. The resulting errors were negligible for the $\mathrm{f}_{0}$ and $\mathrm{f}_{2}$, and contributed a $1.3 \%$ error on the total $\phi$ rate. Since these errors varied with momentum, they are also given in table1 as uncorrelated systematic errors. Because the $\phi$ is close to threshold, there is a small background due to conversion $\mathrm{e}^{+} \mathrm{e}^{-}$pairs, particularly at low $x_{p}$ (as seen in figure 3 ). In the standard fit, this was included in the single background term, $B_{\mathrm{K}^{+} \mathrm{K}^{-}}$of equation (2). When this component was allowed to vary independently, the total $\phi$ rate changed by $2 \%$, which was taken to be the systematic error from this effect. As in [28], an error of $1 \%$ comes from uncertainty in the mass resolution and $1.4 \%$ from modelling of the track cuts. Finally an error of $1.2 \%$ was included for the $\phi \rightarrow \mathrm{K}^{+} \mathrm{K}^{-}$branching ratio, bringing the overall systematic error to $3.5 \%$ (including the contribution from variation of $g_{\mathrm{K}}$ discussed above).

In all of the measurements, the possible presence of other f-meson resonances (for example the $\left.\mathrm{f}_{0}(1360)\right)$ in the $\pi^{+} \pi^{-}$mass spectrum has been neglected. While there is no evidence in the data for such states, and therefore no need to include them in the fits, their presence could in principle give rise to additional systematic errors, particularly for the $\mathrm{f}_{2}$.

\subsection{Production as functions of event multiplicity and rapidity gap}

As discussed in section 1, the Gribov vacuum scalar states are expected to be produced preferentially in low-multiplicity events, and when isolated in rapidity relative to the other particles. Figure 5 shows the fits to the $\pi^{+} \pi^{-}$mass spectra in the $\mathrm{f}_{0}$ mass region for the six bins of event charged-track multiplicity. Figure 6 gives, for each of the three resonances, the ratios of production in data relative to the detector-level JETSET sample (in which the resonances 
are treated as conventional mesons) as a function of the multiplicity. Figure 7 shows the same ratios as a function of the magnitude of the rapidity difference between the resonances and the nearest charged particle. For each resonance the ratios have been normalized such that the weighted averages are unity. No evidence is seen for anomalously large production of $\mathrm{f}_{0}(980)$ either at low multiplicity or at large rapidity gap, with the Monte Carlo model giving a good description. The data therefore do not provide any evidence to support the hypothesis that the $\mathrm{f}_{0}$ is a manifestation of a vacuum scalar state.

\subsection{Production in quark and gluon jets}

The total rates in each of the jets in the selected three-jet events were measured by fitting to the mass spectra in the three bins of scaled energy, and summing the contributions. The jet-finding procedures described in section 3.2 were also applied to the detector-level Monte Carlo sample, and the total number of each of $\mathrm{f}_{0}, \mathrm{f}_{2}$ and $\phi$ in each jet was counted. Figure 8 shows the ratios of total rates measured in the data relative to the detector-level Monte Carlo model for each of the three jet classes. The figure shows no significant evidence for any differences between production in the quark-enriched (high-energy) and the gluon-enriched (low-energy) jets.

\section{Summary and conclusions}

Fragmentation functions and total inclusive rates in $\mathrm{Z}^{0}$ decay have been measured for three resonances, the $\mathrm{f}_{0}(980), \mathrm{f}_{2}(1270)$ and $\phi(1020)$, using the full LEP 1 statistics of OPAL. The $\mathrm{f}_{0}$ and $\mathrm{f}_{2}$ measurements will provide input to understanding the physics of inclusive particle production in the $\mathrm{P}$-wave meson sector, particularly in the context of the Monte Carlo models.

The production characteristics of the $\mathrm{f}_{0}$ show no significant differences from those of the $\mathrm{f}_{2}$ and $\phi$ mesons. In particular, the shapes of the fragmentation functions are similar for all three, and are well reproduced by the JETSET 7.4 Monte Carlo model, within which the $\mathrm{f}_{0}$ is treated as a conventional scalar meson. The total inclusive rate for the $f_{0}$ is $0.141 \pm 0.013$ per hadronic $\mathrm{Z}^{0}$ decay, similar to the value, $0.155 \pm 0.021$, measured for the $\mathrm{f}_{2}$. The $\mathrm{f}_{0}$ rate is significantly larger than for the $\phi, 0.091 \pm 0.004$ per hadronic $\mathrm{Z}^{0}$ decay. These features are consistent with the $\mathrm{f}_{0}$ being, like the $\mathrm{f}_{2}$, a $\mathrm{q} \overline{\mathrm{q}}$ meson in the ${ }^{3} \mathrm{P}$ state composed mainly of $u \bar{u}$ and $\mathrm{d} \overline{\mathrm{d}}$.

For all three states, the production rates relative to the Monte Carlo model have been measured as functions of the charged particle multiplicity of the event and the gap in rapidity to the nearest charged particle. The distributions are found to be flat in all cases. In particular, no evidence has been found for enhanced $\mathrm{f}_{0}$ production at low multiplicities or at large rapidity gap. There is thus no evidence to identify the $\mathrm{f}_{0}$ with the vacuum scalar state proposed by Gribov.

Production in energy-ordered jets in three-jet events has been measured with a view to seeking differences between quark and gluon induced jets. No significant differences are seen between the data and the Monte Carlo model in the relative production rates. There is therefore no evidence for any enhanced gluon content in the $\mathrm{f}_{0}$. 
In summary, all measured characteristics of $\mathrm{f}_{0}(980)$ production in the $\mathrm{Z}^{0}$ decay data of OPAL are consistent with its interpretation as a conventional scalar meson. Quantitative theoretical or phenomenological predictions for production of the types of states discussed in the introduction could enable more definite conclusions to be drawn from the $\mathrm{Z}^{0}$ data.

\section{Acknowledgements}

We particularly wish to thank the SL Division for the efficient operation of the LEP accelerator at all energies and for their continuing close cooperation with our experimental group. We thank our colleagues from CEA, DAPNIA/SPP, CE-Saclay for their efforts over the years on the time-of-flight and trigger systems which we continue to use. In addition to the support staff at our own institutions we are pleased to acknowledge the

Department of Energy, USA, National Science Foundation, USA, Particle Physics and Astronomy Research Council, UK, Natural Sciences and Engineering Research Council, Canada, Israel Science Foundation, administered by the Israel Academy of Science and Humanities, Minerva Gesellschaft, Benoziyo Center for High Energy Physics, Japanese Ministry of Education, Science and Culture (the Monbusho) and a grant under the Monbusho International Science Research Program, German Israeli Bi-national Science Foundation (GIF), Bundesministerium für Bildung, Wissenschaft, Forschung und Technologie, Germany, National Research Council of Canada, Research Corporation, USA, Hungarian Foundation for Scientific Research, OTKA T-016660, T023793 and OTKA F-023259. 


\section{References}

[1] A. Böhrer, Phys. Rep. 291 (1997) 107.

[2] M. R. Pennigton, in Proceedings of the 6th International Conference on Hadron Spectroscopy, Ed. M. C. Birse, G. D. Lafferty and J. A. McGovern, World Scientific (1996).

[3] Particle Data Group, R. M. Barnett al., Phys. Rev. D54 (1996) 1.

[4] R. L. Jaffe and K. Johnson, Phys. Lett. B60 (1976) 201;

R. L. Jaffe, Phys. Rev. D15 (1977) 267 and Phys. Rev. D15 (1977) 269.

[5] J. Weinstein and N. Isgur, Phys. Rev. D41 (1990) 2236.

[6] V. N. Gribov, Lund preprint, LU-TP 91-7.

[7] F. E. Close et al., Phys. Lett. B319 (1993) 291.

[8] S. Ishida et al., in Proceedings of the 6th International Conference on Hadron Spectroscopy, Ed. M. C. Birse, G. D. Lafferty and J. A. McGovern, World Scientific (1996);

S. Ishida, H. Sawazaki, M. Oda and K. Yamada, Phys. Rev. D47 (1993) 179.

[9] D. Robson, N. Phys. B130 (1977) 328.

[10] C. Amsler and F. E. Close, Phys. Lett. B353 (1995) 385.

[11] G. Bali et al., Phys. Lett. B307 (1993) 378;

D. Weingarten et al., N. Phys. B34 (1994) 29.

[12] N. A. Törnqvist et al., Z. Phys. C68 (1995) 647.

[13] B. S. Zou and D. V. Bugg, Phys. Rev. D48 (1993) R3948.

[14] A. V. Anisovich and A. V. Sarantsev, Phys. Lett. B413 (1997) 137.

[15] T. Sjöstrand, Comp. Phys. Commun. 82 (1994) 74.

[16] B. Andersson, G. Gustafson, G. Ingelman and T. Sjöstrand, Phys. Rep. 97 (1983) 31.

[17] I. G. Knowles and G. D. Lafferty, J. Phys. G: Nucl. Part. Phys. 23 (1997) 731.

[18] DELPHI Coll., P. Abreu et al., Z. Phys. C65 (1994) 587.

[19] ALEPH Coll., D. Buskulic et al., Z. Phys. C69 (1995) 379.

[20] OPAL Coll., K. Ahmet et al., Nucl. Instrum. Methods A305 (1991) 275.

[21] P.P. Allport et al., Nucl. Instrum. Methods A324 (1993) 34;

P.P. Allport et al., Nucl. Instrum. Methods A346 (1994) 476.

[22] M. Hauschild et al., Nucl. Instrum. Methods A314 (1992) 74.

[23] J. Allison et al., Nucl. Instrum. Methods A317 (1992) 47. 
[24] OPAL Coll., M.Z. Akrawy et al., Z. Phys. C47 (1990) 505;

The JETSET 7.4 parameters were tuned as described in OPAL Coll., G. Alexander et al., Z. Phys. C69 (1996) 543.

[25] OPAL Coll., G. Alexander at al., Z. Phys. C5 (1991) 175.

[26] J. B. Gay et al., Phys. Lett. 63B (1976) 220;

S. M. Flatté, Phys. Lett. 63B (1976) 224 and Phys. Lett. 63B (1976) 228.

[27] S. Catani, Yu. L. Dokshitzer, F. Fiorani and B. R. Webber, N. Phys. B377 (1992) 445;

S. Catani et al., Phys. Lett. B269 (1991) 432;

S. Bethke, Z. Kunszt, D. E. Soper and W. J. Stirling, N. Phys. B370 (1992) 310;

N. Brown and W. J. Stirling, Z. Phys. C53 (1992) 629.

[28] OPAL Coll., R. Akers et al., Z. Phys. C68 (1995) 1.

[29] ALEPH Coll., D. Buskulic et al., Z. Phys. C69 (1996) 379.

[30] DELPHI Coll., P. Abreu et al., Z. Phys. C73 (1996) 61.

[31] G. D. Lafferty and T. R. Wyatt, Nucl. Instrum. Methods A355 (1995) 541. 


\begin{tabular}{|c|c|c|c|}
\hline$x_{p}$ range & $\mathrm{f}_{0}(980)$ & $\mathrm{f}_{2}(1270)$ & $\phi(1020)$ \\
\hline $0.00-0.06$ & $1.04 \pm 0.09$ & $1.00 \pm 0.14$ & $0.464 \pm 0.011 \pm 0.005$ \\
$0.06-0.12$ & $0.57 \pm 0.05$ & $0.69 \pm 0.08$ & $0.316 \pm 0.021 \pm 0.007$ \\
$0.12-0.14$ & $0.30 \pm 0.06$ & $0.41 \pm 0.09$ & $0.285 \pm 0.020 \pm 0.009$ \\
$0.14-0.16$ & $0.20 \pm 0.05$ & $0.25 \pm 0.08$ & $0.197 \pm 0.019 \pm 0.006$ \\
$0.16-0.20$ & $0.21 \pm 0.03$ & $0.27 \pm 0.04$ & $0.167 \pm 0.017 \pm 0.002$ \\
$0.20-0.25$ & $0.13 \pm 0.02$ & $0.22 \pm 0.03$ & $0.133 \pm 0.007 \pm 0.002$ \\
$0.25-0.35$ & $0.085 \pm 0.011$ & $0.091 \pm 0.016$ & $0.096 \pm 0.004 \pm 0.001$ \\
$0.35-0.50$ & $0.046 \pm 0.005$ & $0.035 \pm 0.008$ & $0.045 \pm 0.002 \pm 0.001$ \\
$0.50-1.00$ & $0.0079 \pm 0.0009$ & $0.008 \pm 0.001$ & $0.010 \pm 0.001 \pm 0.000$ \\
\hline $0.00-1.00$ & $0.141 \pm 0.007$ & $0.155 \pm 0.011$ & $0.091 \pm 0.002 \pm 0.001$ \\
\hline
\end{tabular}

Table 1: Measured differential cross sections, $\left(1 / \sigma_{\mathrm{h}}\right) \mathrm{d} \sigma / \mathrm{d} x_{p}$, and total inclusive rates for $\mathrm{f}_{0}$, $\mathrm{f}_{2}$ and $\phi$ production. There are additional overall systematic errors of $7.6 \%$ for the $\mathrm{f}_{0}, 11.6 \%$ for the $\mathrm{f}_{2}$ and $3.5 \%$ for the $\phi$. The uncorrelated systematic errors for the $\phi$ are given in the table; the error for the $x_{p}$ range $0.50-1.00$ is smaller than 0.0005 . The uncorrelated systematic errors for the $\mathrm{f}_{0}$ and $\mathrm{f}_{2}$ are negligible in comparison with the statistical errors and the correlated systematic errors.

\begin{tabular}{|c|c|c|c|}
\hline & $\mathrm{f}_{0}(980)$ & $\mathrm{f}_{2}(1270)$ & $\phi(1020)$ \\
\hline Variation of $m_{0}$ & $2.5 \%$ & - & - \\
\hline Variation of $g_{\pi}$ & $7.0 \%$ & - & - \\
\hline Variation of $g_{\mathrm{K}}$ & - & - & $1.5 \%$ \\
\hline $\mathrm{f}_{2}$ resonance line shape & - & $8.7 \%$ & - \\
\hline $\mathrm{f}_{2}$ mass and width & - & $6.0 \%$ & - \\
\hline $\mathrm{BR}\left(\mathrm{f}_{2} \rightarrow \pi^{+} \pi^{-}\right)$ & - & $3.1 \%$ & - \\
\hline$\rho^{0}$ resonance line shape & - & $3.2 \%$ & - \\
\hline $\mathrm{d} E / \mathrm{d} x$ parametrization & - & - & $1.3 \%$ \\
\hline $\mathrm{BR}\left(\phi \rightarrow \mathrm{K}^{+} \mathrm{K}^{-}\right)$ & - & - & $1.2 \%$ \\
\hline $\mathrm{K}^{+} \mathrm{K}^{-}$mass resolution & - & - & $1.0 \%$ \\
\hline Photon conversions & - & - & $2.0 \%$ \\
\hline Track cuts & $1.4 \%$ & $1.4 \%$ & $1.4 \%$ \\
\hline Total & $7.6 \%$ & $11.6 \%$ & $3.5 \%$ \\
\hline
\end{tabular}

Table 2: Sources of systematic errors on the total inclusive meson rates. Errors on the $\phi$ rates from uncertainties in the parametrization of $\mathrm{d} E / \mathrm{d} x$ are momentum dependent, and are given in table 1 for the bins of $x_{p}$. 


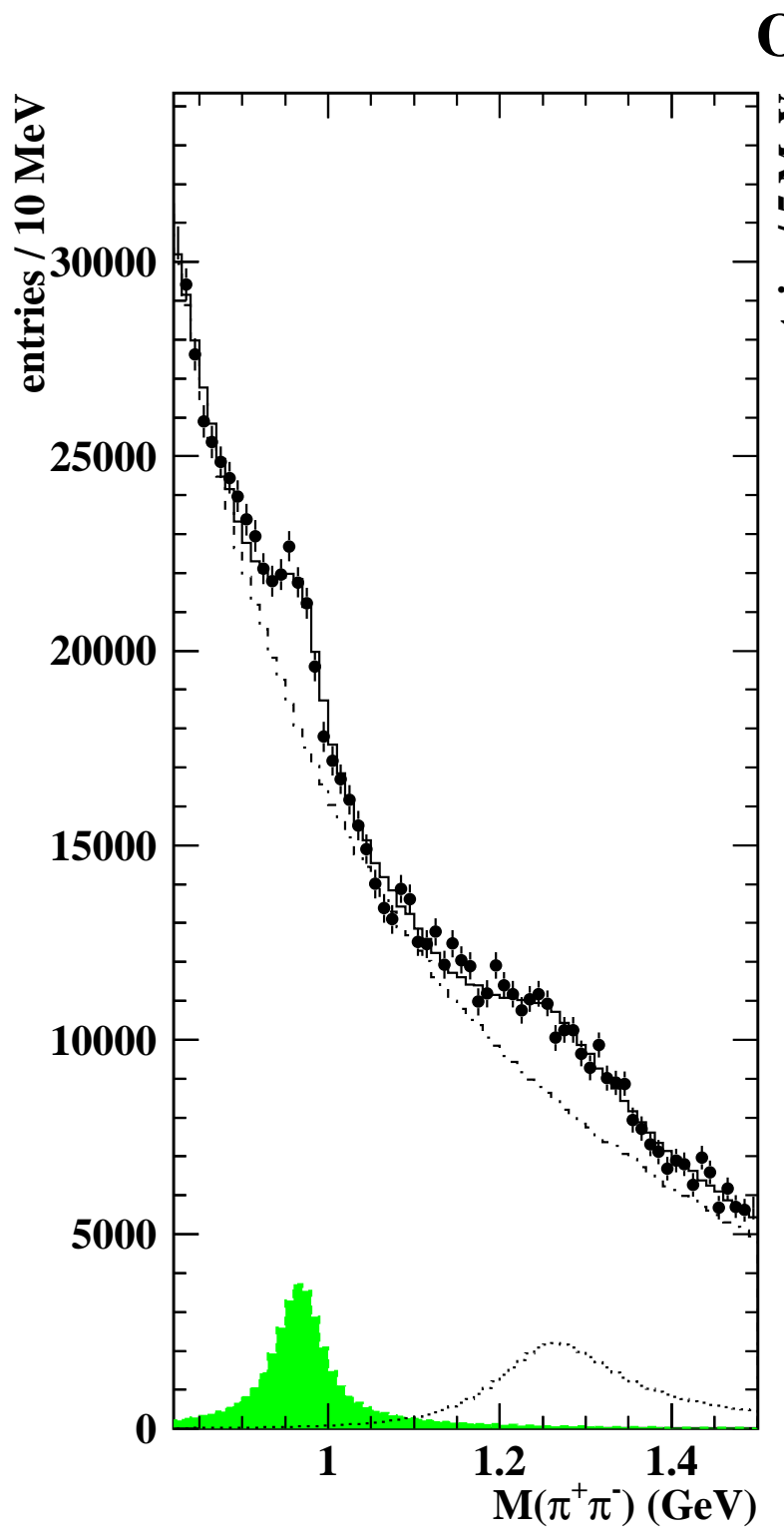

OPAL

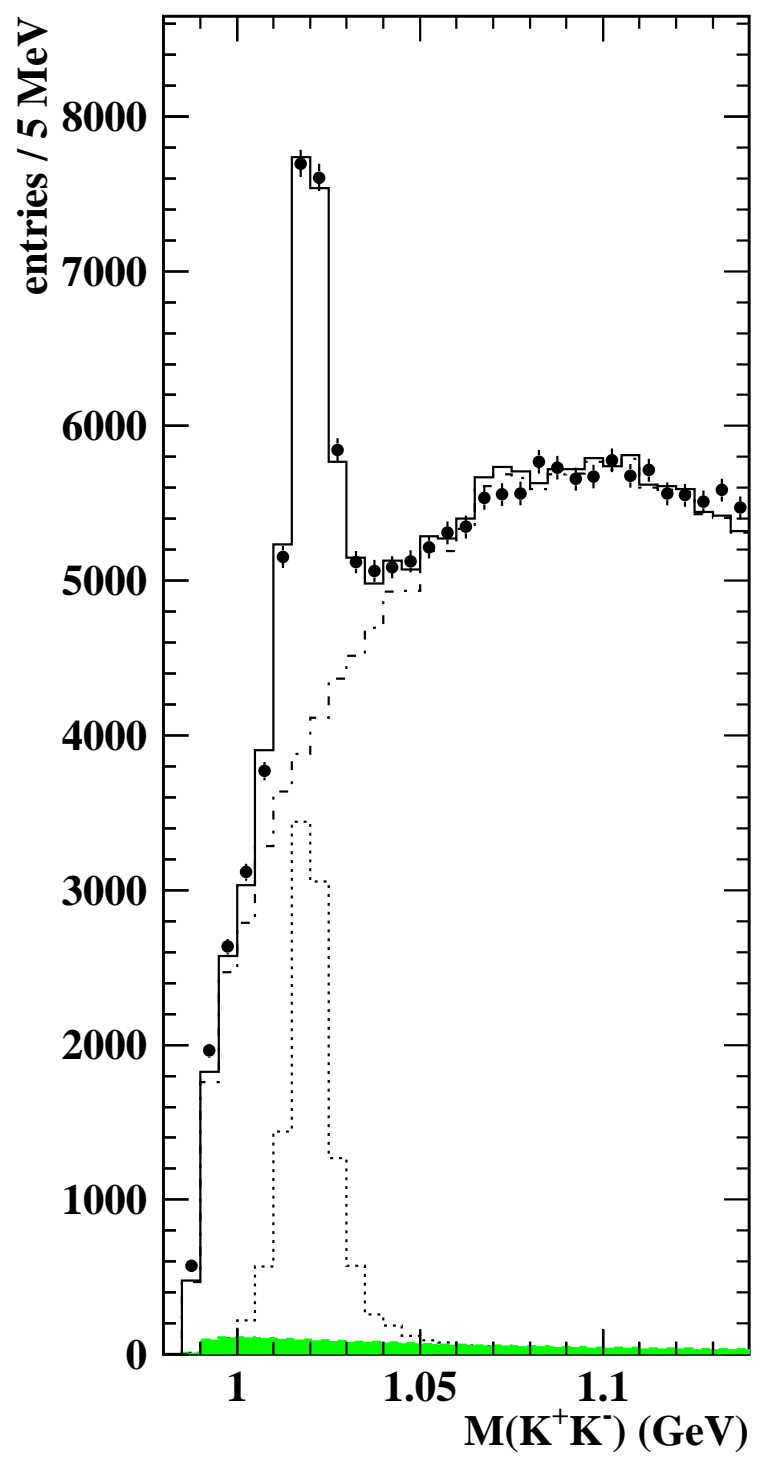

Figure 1: Sum of the fitted mass spectra of $\pi^{+} \pi^{-}$(with like-sign spectra subtracted) and $\mathrm{K}^{+} \mathrm{K}^{-}$ for $x_{p}>0.14$ with the results of the fits described in the text. The points show the data, and the solid histograms give the results of the fits. The shaded histograms show the $\mathrm{f}_{0}$ contributions, the dotted histograms give the $\mathrm{f}_{2}$ and $\phi$ contributions, and the dot-dash histograms show the fitted backgrounds. 


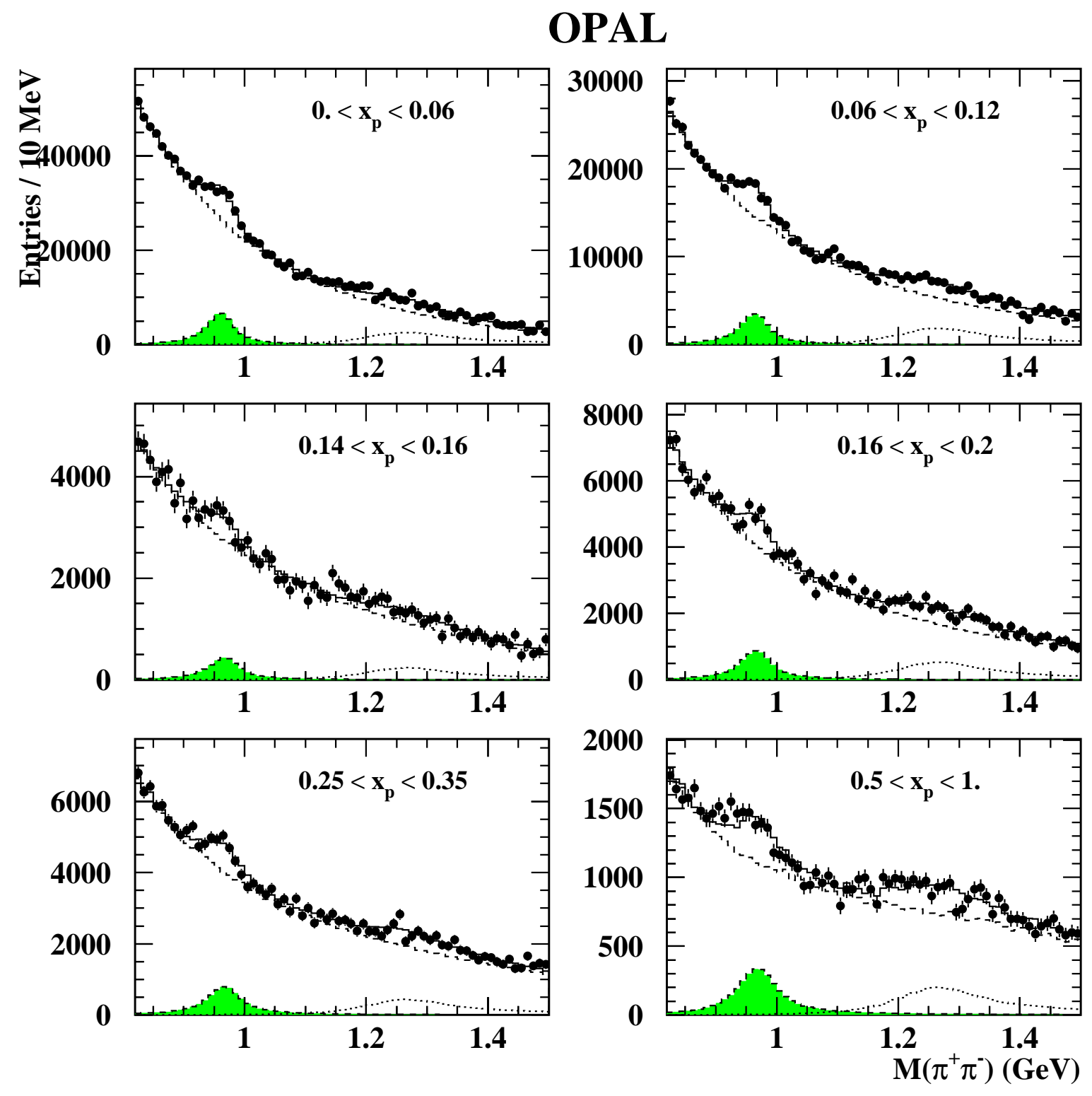

Figure 2: Mass spectra of $\pi^{+} \pi^{-}$(with like-sign spectra subtracted) for 6 bins of $x_{p}$ with the results of the fits described in the text. The points show the data, and the solid histograms give the results of the fits. The shaded histograms show the $\mathrm{f}_{0}$ contributions, the dotted histograms show the $\mathrm{f}_{2}$ contributions, and the dot-dash histograms show the fitted backgrounds. 


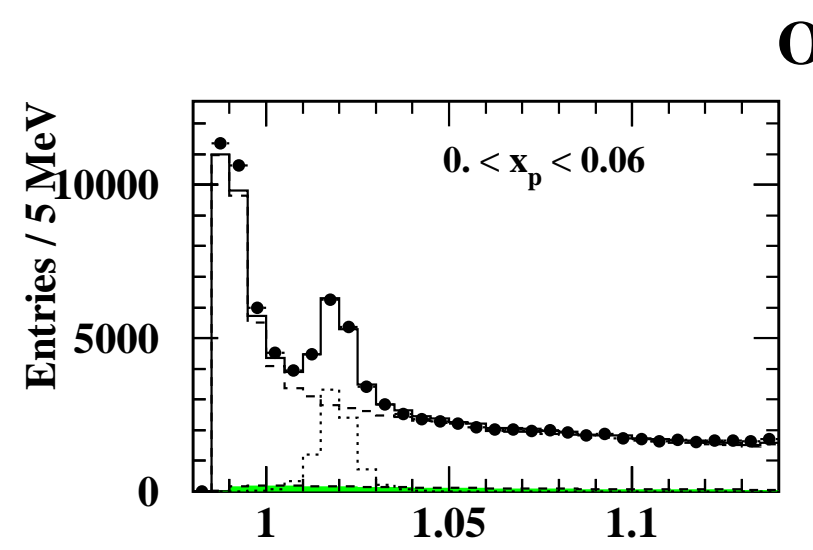

OPAL
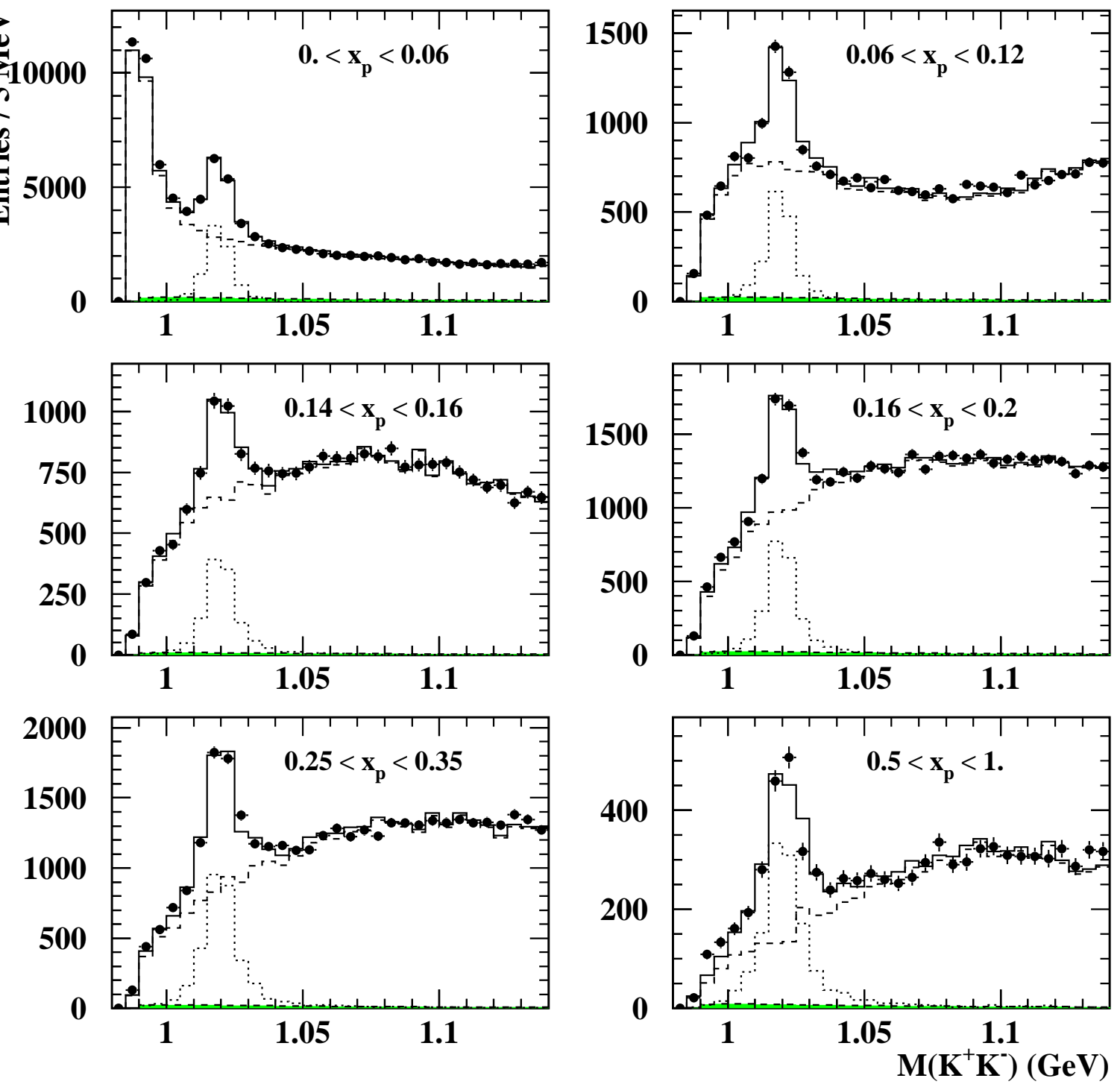

Figure 3: Mass spectra of $\mathrm{K}^{+} \mathrm{K}^{-}$for 6 bins of $x_{p}$ with the results of the fits described in the text. The points show the data, and the solid histograms give the results of the fits. The shaded histograms show the $\mathrm{f}_{0}$ contributions, the dotted histograms show the $\phi$ contributions, and the dot-dash histograms show the fitted backgrounds. 


\section{OPAL}

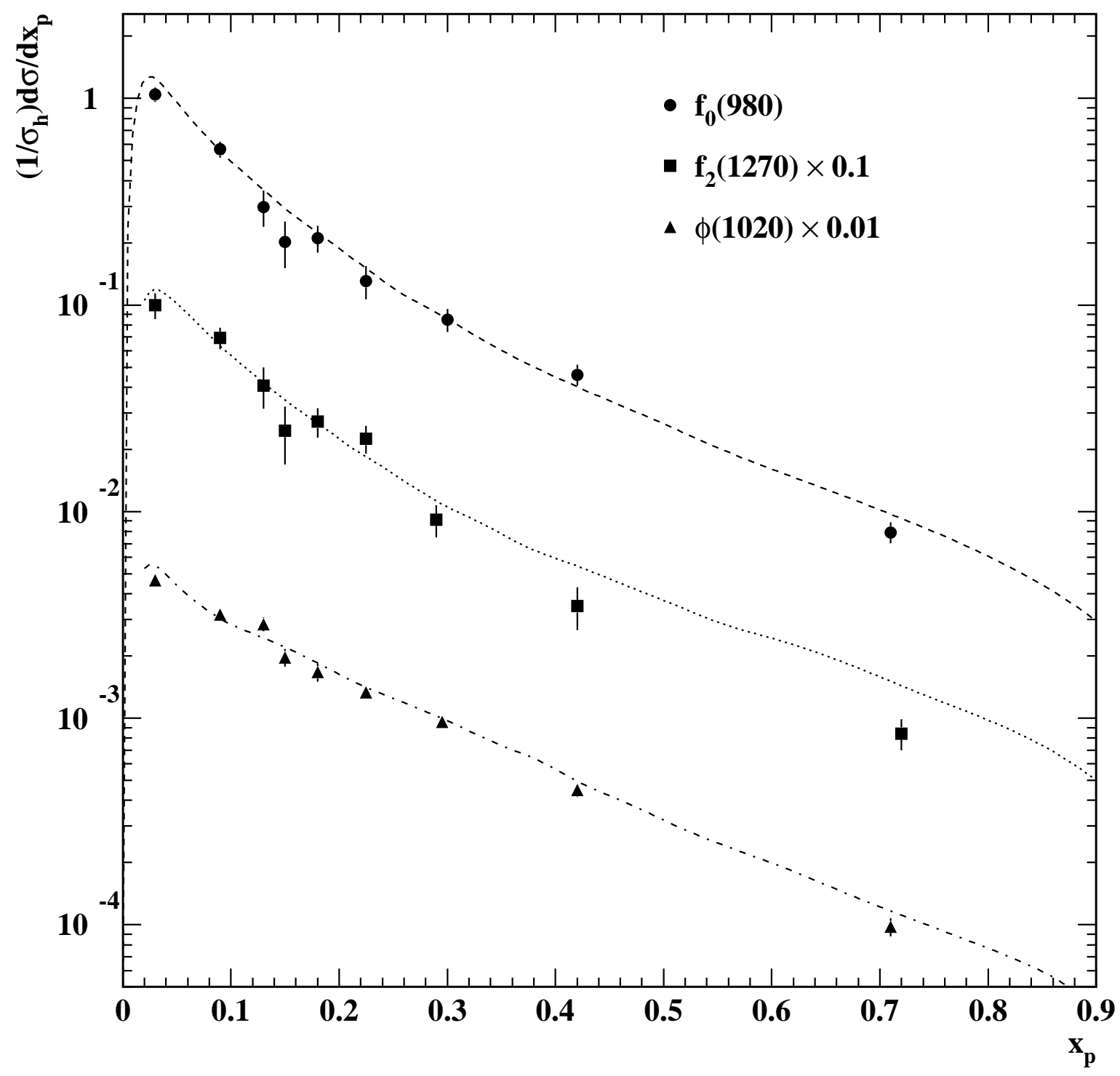

Figure 4: Measured fragmentation functions for $\mathrm{f}_{0}, \mathrm{f}_{2}$ and $\phi$ together with the output of the JETSET 7.4 generator. The $\mathrm{f}_{2}$ and $\phi$ measurements have been scaled by $\times 0.1$ and $\times 0.01$ respectively. The Monte Carlo curves have been normalized to the same total rate as in the data. For $\mathrm{f}_{0}$ and $\mathrm{f}_{2}$, the errors are statistical only. For the $\phi$, the uncorrelated systematic errors are included. There are additional, fully correlated, systematic errors of $7.6 \%$ for the $\mathrm{f}_{0}, 11.6 \%$ for the $\mathrm{f}_{2}$ and $3.5 \%$ for the $\phi$. The $\mathrm{x}$-coordinates for plotting the data points were evaluated following [31]. 


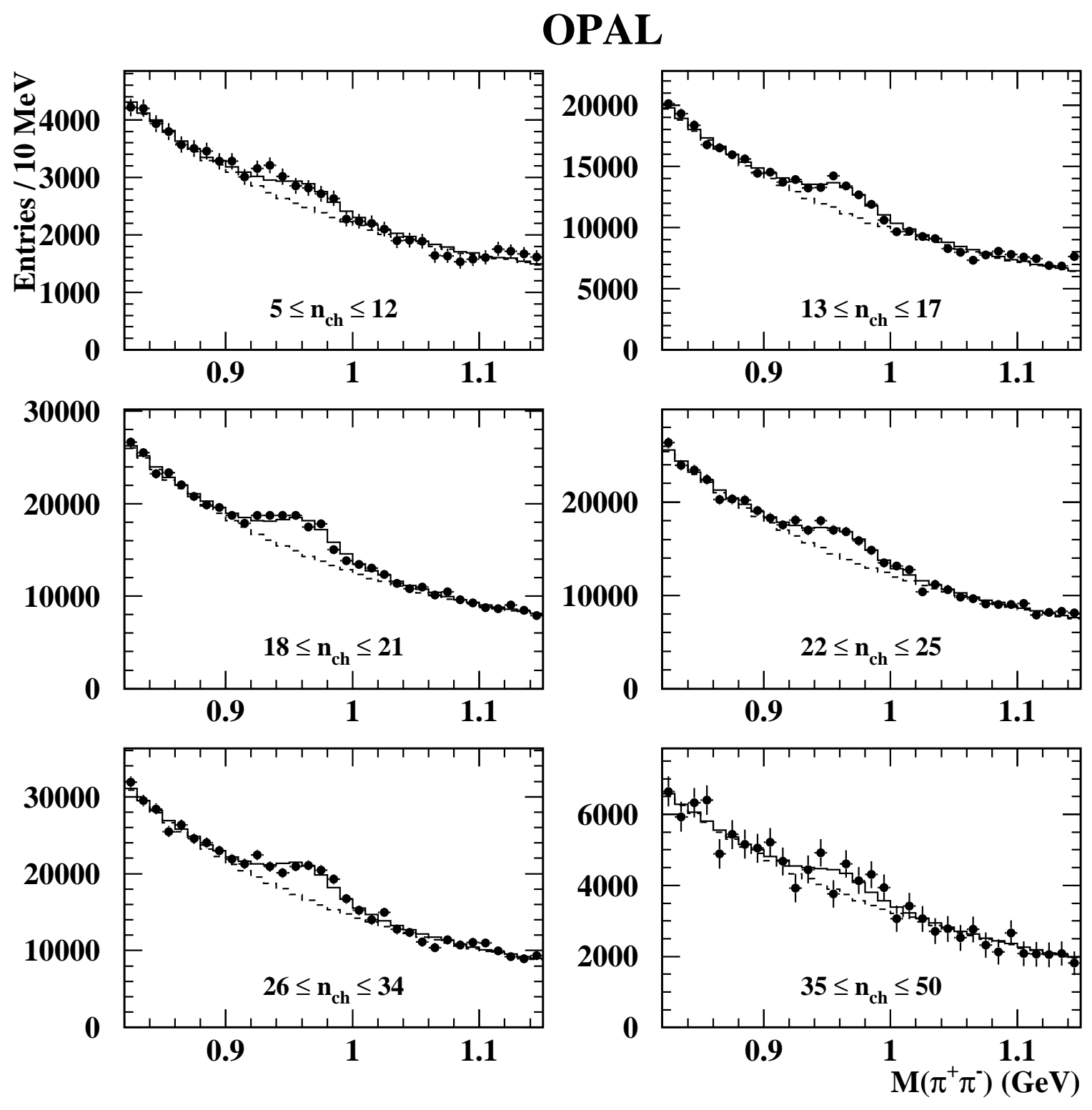

Figure 5: Fitted $\pi^{+} \pi^{-}$spectra (with like-sign spectra subtracted) in the $\mathrm{f}_{0}$ mass region for bins of event charged-particle multiplicity. The points show the data, and the solid histograms give the results of the fits. The dashed histograms show the fitted backgrounds. 


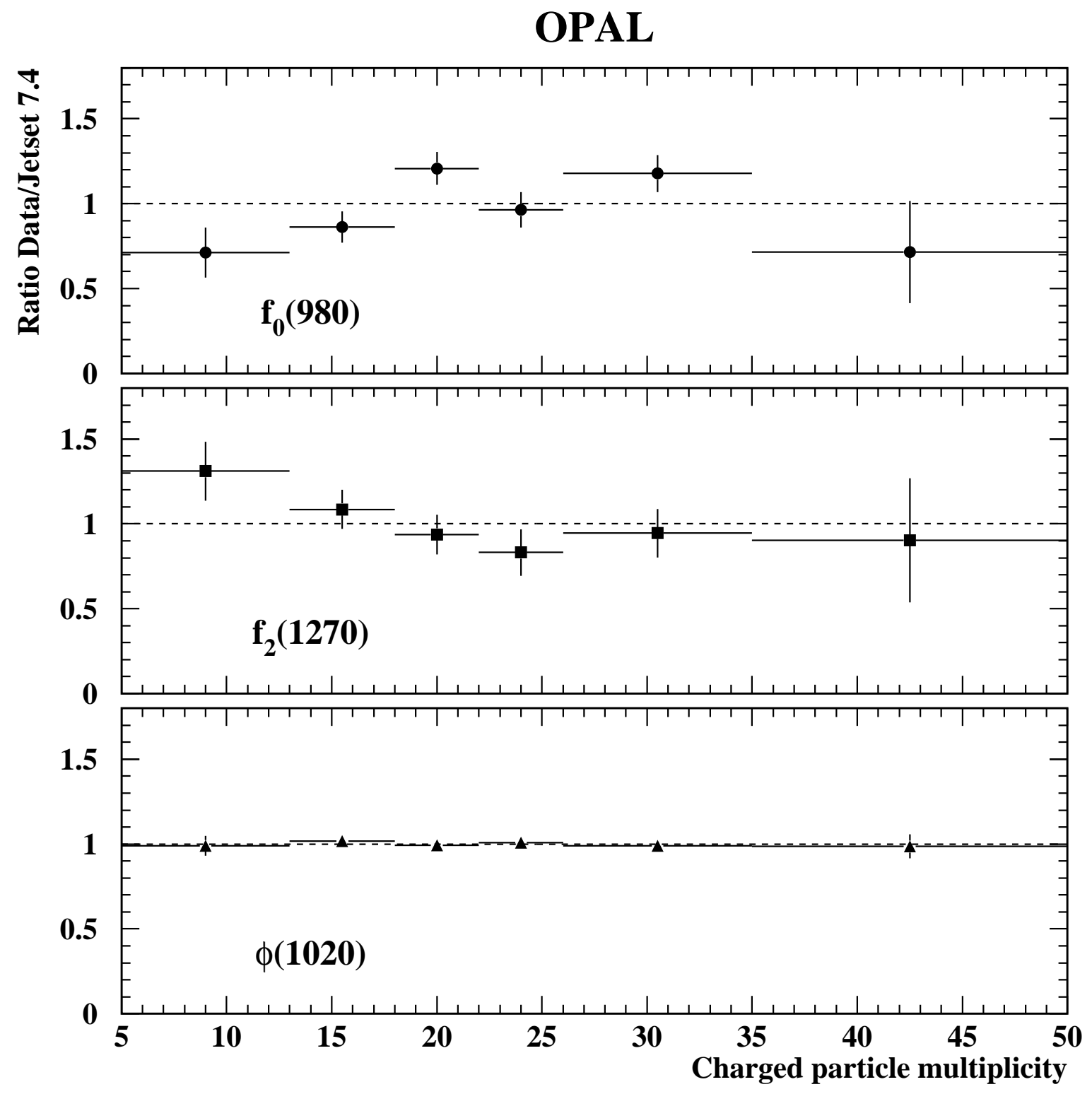

Figure 6: Ratios of production rates in data compared to the detector-level JETSET 7.4 model, for bins of event charged-particle multiplicity. The errors are statistical only. The ratios have been normalized such that the weighted average is unity for each particle. 


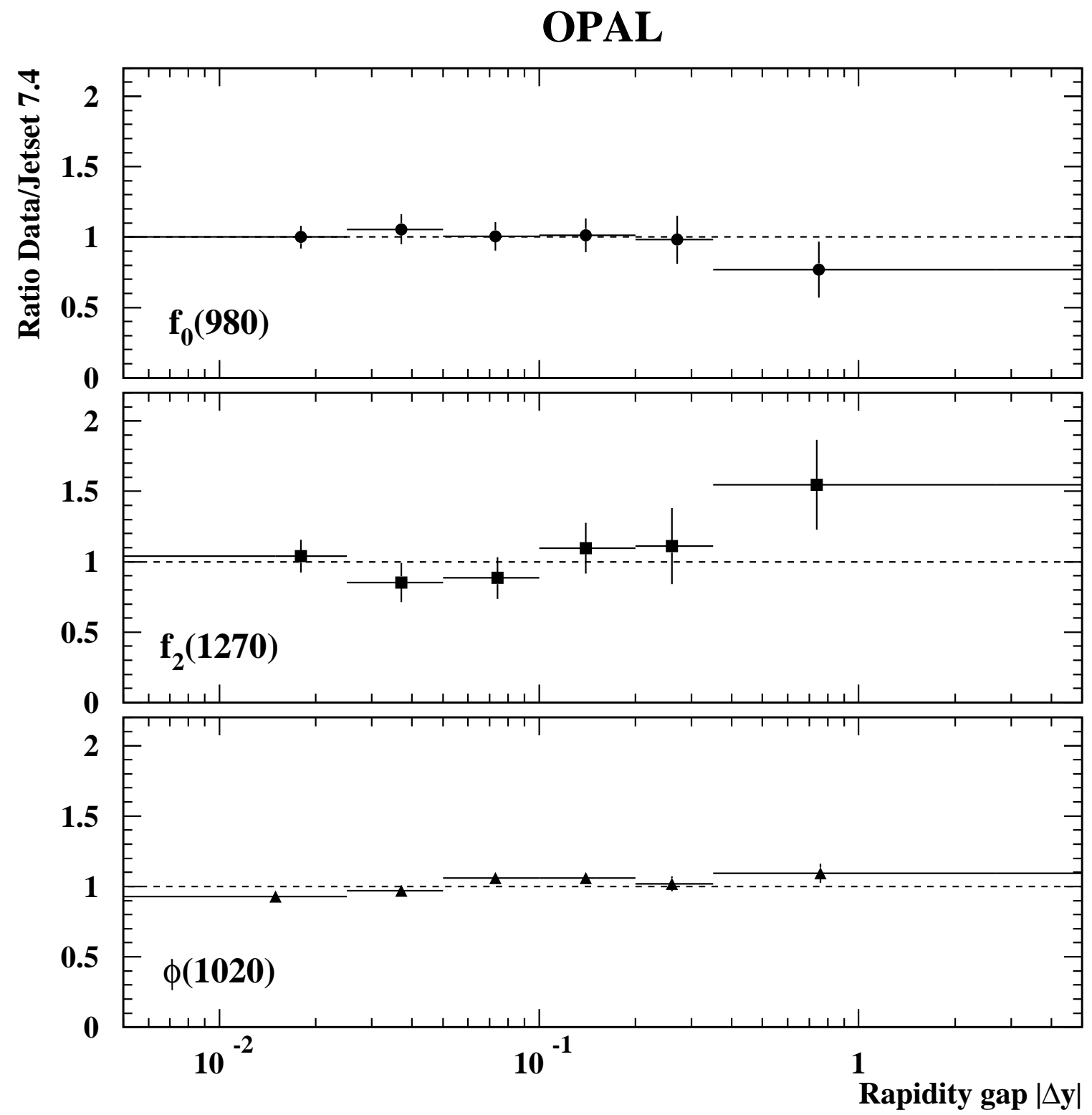

Figure 7: Ratios of total production rates in data compared to the detector-level JETSET 7.4 model, for bins of the absolute value of the rapidity difference between the meson and the nearest charged particle. The errors are statistical only. The ratios have been normalized such that the weighted average is unity for each particle. The lowest bin extends down to zero, while the highest extends to the kinematic limit. 


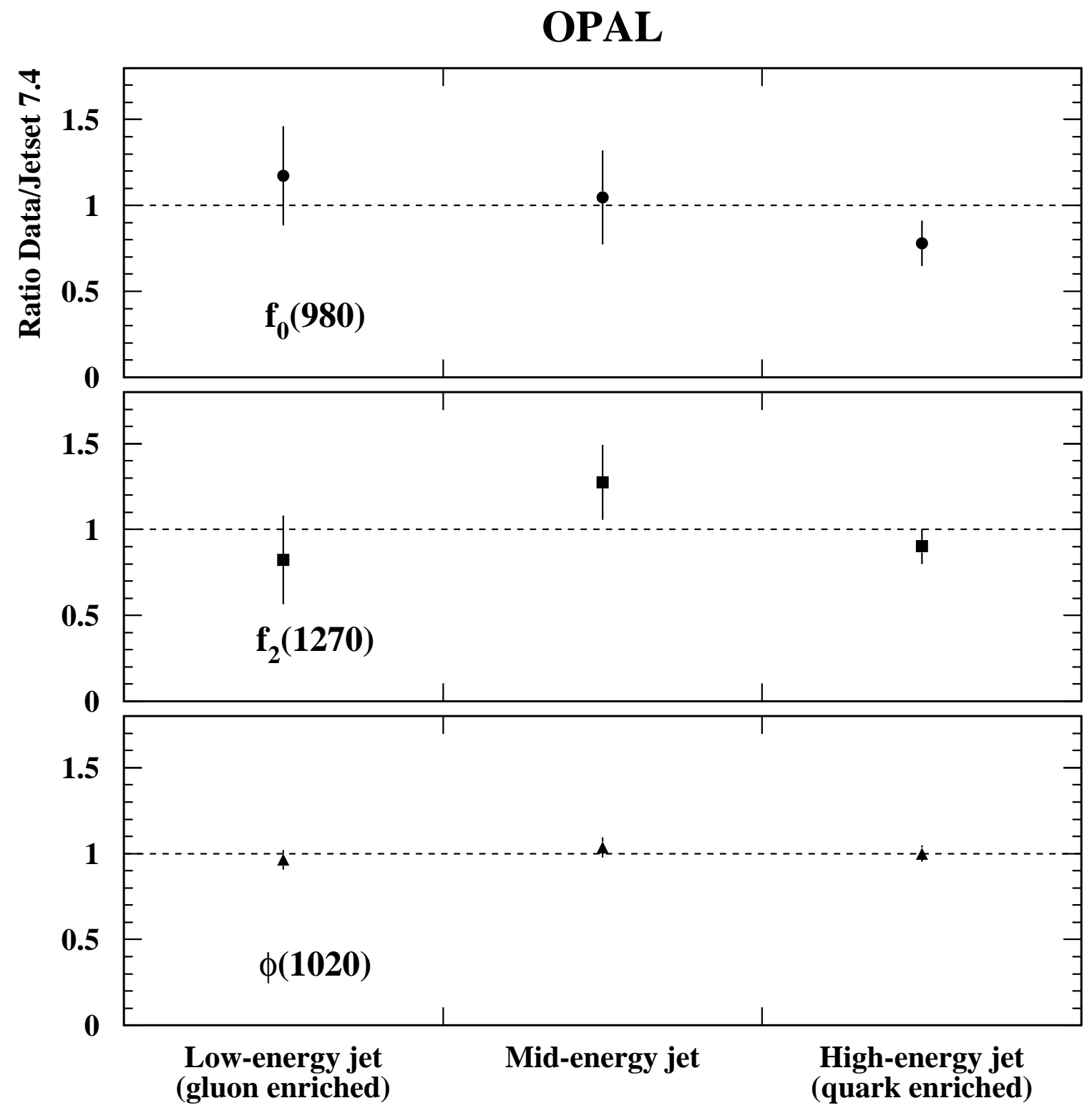

Figure 8: Ratios of total production rates in data compared to the detector-level JETSET 7.4 model for the energy-ordered jets in three-jet events. The errors are statistical only. The ratios have been normalized for each particle to give a weighted average value of unity over the three jets. 